\title{
A Spin Polarized Disc. II
}

\author{
J. P. Krisch ${ }^{1}$ and L. L. Smalley ${ }^{2}$
}

Received April 19, 1995

\begin{abstract}
We present a solution to the gravitational field equations which describes either a static disc in general relativity or a spin polarized rotating disc in a Riemann-Cartan spacetime. The disc has infinite radius and finite thickness. The energy and pressure are finite and positive within the disc and for some parameter choices, a radial fall off in the fluid parameters is possible. A comparison is made to axis-symmetric wall solutions.
\end{abstract}

\section{INTRODUCTION}

Disc shaped mass distributions have been frequently used to model galactic structures. Since many galaxies are highly compressed in the $z$-direction compared to their radial extent, the simple static models often assume that the galactic volume density is a function only of $z$. A model of this type was introduced by Oort [1]. It is a simple infinite slab of collisionless matter running between $z= \pm H$ with a density $\rho_{0}(z)$ and a gravitational potential [2]

$$
\Phi=\frac{4 \pi G \rho_{0} z^{2}}{2}+\text { constant }
$$

$\pm H$ are the boundaries of the disc. Discs containing matter under pressure have also been discussed in a classical background [3]. Models of

1 Department of Physics, The University of Michigan, Ann Arbor, Michigan 48109, USA

2 Department of Physics, University of Alabama in Hunstville, Huntsville, Alabama 35899, USA 
self-similar discs have been developed by Lynden-Bell and Pineault [4] and Lemos [5]. Finite discs were treated by Morgan and Morgan [6] who also discussed counterrotating discs. Bičak, Lynden-Bell and Katz [7] used counterrotating discs as sources for static vacua and Bičak and Ledvinka [8] have applied this to the Kerr vacuum. Kuzmin [9] has discussed the Newtonian gravitational field of a thin disc and his work was extended by Evans and deZeeuw [10]. In this note we describe a relativistic, infinite disc solution with a metric structure that, for small $z$, generates a potential similar to eq. (1). The matter content of the disc is a fluid under pressure and it can be interpreted in two ways. It is either (i) a static anisotropic fluid in a general relativistic spacetime or it is (ii) an axially polarized rotating spin-fluid in a Riemann-Cartan spacetime. The formalism that shows the equivalence of the two descriptions was developed by Krisch and Smalley [11]. For either interpretation, the fluid parameters are well behaved. This paper is the second [12] in a series that discusses spin polarized disc solutions. Discs have been used frequently in other calculations concerned only with the surface stress energy content of the source [13-18]. The solution presented in this note has a well-defined and simple, if anisotropic, interior stress energy content. The solution may be useful in modeling large rotating structures with embedded intrinsic angular momentum. In addition, infinite discs with specific symmetries have been used to discuss wall defects [19-23]. This aspect of our solution will be discussed at the end of the paper.

\section{METRIC AND MATTER CONTENT}

\section{A. Metric}

We wish to consider the field equations for a spacetime with metric

$$
d s^{2}=-f\left(d t+\frac{k}{f} d \phi\right)^{2}+\exp (\mu) d r^{2}+\exp (\beta) d z^{2}+\frac{D^{2}}{f} d \phi^{2} .
$$

The form that we will assume for the metric potentials is

$$
\begin{aligned}
f & =\left(r+r_{0}\right)^{d} F(z) \\
k & =r^{g} G(z) \\
D & =\left(r+r_{0}\right)^{s} D(z) \\
\exp (\mu) & =\left(r+r_{0}\right)^{b-2} \exp [\mu(z)] \\
\exp (\beta) & =\left(r+r_{0}\right)^{b} \exp [\mu(z)] .
\end{aligned}
$$


The solutions that we generate will describe both a static general relativistic fluid and a stationary spin fluid in a spacetime with torsion. This form for the metric has been used previously in a discussion of the energy conditions in a spacetime with torsion [24]. $F(z)$ will be assumed to be

$$
F(z)=D^{2 h}(z), \quad D^{2}(z)=\exp \left[\alpha z^{2}\right] .
$$

Since, in the Newtonian limit, $g_{00}=1+2 \Phi$, this choice will produce the quadratic potential of the simple disc models, eq. (1). Other metric choices could have been made that also provided a quadratic potential. The choice $(3),(4)$ is convenient because of the existing discussions of energy density interpretation.

In our notation, we denote the coordinates $(t, r, z, \phi)$ by $(0,1,2,3)$ and prime $\left({ }^{\prime}\right)$ denotes derivative with respect to $z$. It is convenient to introduce the tetrads that diagonalize the metric:

$$
\begin{aligned}
e^{\nu}(0) & =\left(\frac{1}{\sqrt{f}}, 0,0,0\right) \\
e^{\nu}(1) & =\left(0, \exp \left[\frac{-\mu(z)}{2}\right], 0,0\right) \\
e^{\nu}(2) & =\left(0,0, \exp \left[\frac{-\beta(z)}{2}\right], 0\right) \\
e^{\nu}(3) & =\left(\frac{-k}{\sqrt{f} D}, 0,0, \frac{\sqrt{f}}{D}\right) .
\end{aligned}
$$

These tetrads will be used to index the field equations

The spacetime contains a spin fluid - a perfect fluid with a spin density, $S_{i j}$, defined throughout its extent. The stress energy tensor for this kind of fluid has been developed and discussed extensively by Ray and Smalley [25]. Using cylindrical coordinates $(r, \phi, z)$ we have three possible spin densities $S_{r \phi}, S_{z \phi}, S_{r z}$. We wish to develop a solution describing a disc with a axially polarized spin content; $S_{r \phi}$ will be the only non-zero spin density. $S_{r z}$ is non-zero only if $f$ and $k$ are directly proportional, i.e. the fluid is completely irrotational in general relativity. We will avoid this case. Zeroing $S_{z \phi}$ will constrain the metric potentials. The stresses and energy density developed for the Riemann-Cartan spin fluid will be the same as for the zero spin static general relativistic solution.

B. Spin-metric constraints. The off diagonal field equations

We will focus on the particular case where the spin densities $S_{r \phi}$ and $S_{z \phi}$ are proportional to the classical fluid vorticities.

$$
S_{r \phi}=\frac{f k_{r}-k f_{r}}{f \sqrt{f}}=\frac{k}{\sqrt{f}}\left(\frac{g}{r}-\frac{d}{r+r_{0}}\right)
$$




$$
S_{z \phi}=\frac{f k_{z}-k f_{z}}{f \sqrt{f}}=\frac{k}{\sqrt{f}}\left(\frac{F^{\prime}}{F}-\frac{G^{\prime}}{G}\right)
$$

Requiring $S_{z \phi}$ to be zero imposes the constraint

$$
\frac{F^{\prime}}{F}=\frac{G^{\prime}}{G}
$$

so that

$$
G(z)=c_{1} F(z)=c_{1} \exp \left[h \alpha z^{2}\right]
$$

with $c_{1}$ an integration constant.

Requiring the spin density to be proportional to the vorticity creates a special type of solution to the field equations in a Riemann-Cartan (RC) spacetime. It has been shown that with the relations $(6),(7)$, the diagonal and $r z$ field equations are formally identical to the field equations for a static general relativistic perfect fluid [11]. The remaining (03) field equation is used to determine the off diagonal metric component in the RC spacetime. The solutions that we find will be valid for either a static perfect fluid in general relativity or a stationary spin fluid in an RC spacetime. The (tetrad indexed) (03) equation constrains the metric potentials.

$$
\text { (03): } \quad\left(r+r_{0}\right)\left(\frac{g}{r}-\frac{d}{r+r_{0}}\right) d=\frac{F^{\prime}(z)}{F(z)}\left(\frac{F^{\prime}(z)}{F(z)}-\frac{G^{\prime}(z)}{G(z)}\right) \text {. }
$$

If $S_{z \phi}$ is to be zero and $S_{r \phi}$ non-zero we must have

$$
d=0 \text {. }
$$

The $r z$ field equation provides further constraining relations on the metric functions.

$$
r z: \quad \mu^{\prime}(z) s+(b-2 s) \frac{D^{\prime}}{D}+s \frac{F^{\prime}}{F}=0 .
$$

There are three possibilities,

$$
\begin{aligned}
& \text { (a) } s=0, b=0, \\
& \text { (b) } s=0, D^{\prime}=0, \\
& \text { (c) } s \neq 0, \mu^{\prime}(z)+\frac{F^{\prime}}{F}=\frac{2 s-b}{s} \frac{D^{\prime}}{D} .
\end{aligned}
$$


Case (a) was considered in an earlier paper [12]. Case (b) produces unphysical fluid parameters. The spin polarized discs that are the subject of this paper occur for case (c). Using eq. (14) we have

$$
\mu(z)=\left(\frac{2 s-b}{s}-2 h\right) \frac{a z^{2}}{2} .
$$

The integration constant has been scaled into the coordinate definition.

The form of the metric is

$$
\begin{aligned}
d s^{2}= & -\exp \left[h \alpha z^{2}\right]\left\{d t+c_{1} r^{g} d \phi\right\}^{2}+\exp \left[\alpha z^{2}(1-h)\right]\left(r+r_{0}\right)^{2 s} d \phi^{2} \\
& +\exp \left[\left(\frac{2 s-b}{s}-2 h\right) \frac{\alpha z^{2}}{2}\right]\left(r+r_{0}\right)^{b-2}\left\{d r^{2}+\left(r+r_{0}\right)^{2} d z^{2}\right\}
\end{aligned}
$$

For the static general relativistic metric, $c_{1}=0 . c_{1} \neq 0$ is the EinsteinCartan case.

C. Stress energy content. The diagonal field equations

The diagonal field equations generate the pressure and energy density. For case (c) the diagonal equations generating the pressure and energy density are

$$
\begin{aligned}
& \exp [\mu(z)]\left(r+r_{0}\right)^{b} p_{1}=\frac{b s}{2}+\alpha+\alpha^{2} z^{2}\left(h^{2}+1+\frac{b-2 s}{2 s}\right), \\
& \exp [\mu(z)]\left(r+r_{0}\right)^{b} p_{2}=s \frac{2 s-b}{2}+\alpha^{2} z^{2}\left(\frac{2 s-b}{2 s}-h^{2}\right), \\
& \exp [\mu(z)]\left(r+r_{0}\right)^{b} p_{3}=\alpha \frac{2 s-b}{2 s}+h^{2} \alpha^{2} z^{2}, \\
& \exp [\mu(z)]\left(r+r_{0}\right)^{b} \epsilon=-s^{2}+\alpha\left(2 h-1+\frac{b-2 s}{2 s}\right)-(h-1)^{2} \alpha^{2} z^{2} .
\end{aligned}
$$

\section{Spin content}

Equations (17)-(20) refer to either a static disc in general relativity or a rotating disc in a Riemann-Cartan spacetime. If the space is RiemannCartan than the spin density of the fluid in the disc is

$$
S_{r \phi}=\frac{f k_{r}-k f_{r}}{f \sqrt{f}}=c_{1} \exp \left[\frac{h a z^{2}}{2}\right] g r^{g-1} .
$$

$g>1$ will guarantee that the vorticity and the spin vanish on the axis of rotation. 
E. The $z$ cutoff for the disc

The upper and lower surfaces of the disc can be defined as the surfaces over which $p_{2}$ vanishes. Using eq. (18) we find for a non-zero denominator

$$
\alpha H= \pm \sqrt{\frac{[s(2 s-b) / 2]}{h^{2}-[(2 s-b) / 2 s]}}
$$

If $h=0$ and $b=2 s$, the $z$ pressure is identically zero and the boundary must be identified from a matching procedure. If there is no matching the pressure becomes a tension. The case $s=0$ was discussed previously [12].

\section{PARAMETER CONSTRAINTS}

Requirements on the sign and finiteness of the energy and pressure set parameter constraints. (We do not consider acoustic limits.) First look at the disc in the $(r, \phi)$ plane, $z=0$. The fluid parameters in this central disc are

$$
\begin{aligned}
& \left(r+r_{0}\right)^{b} p_{1}=\frac{b s}{2}+\alpha, \\
& \left(r+r_{0}\right)^{b} p_{2}=s \frac{2 s-b}{2} \\
& \left(r+r_{0}\right)^{b} p_{3}=\alpha \frac{2 s-b}{2 s} \\
& \left(r+r_{0}\right)^{b} \epsilon=-s^{2}+\alpha\left(2 h-1+\frac{b-2 s}{2 s}\right) .
\end{aligned}
$$

Assuming $s>0$ and $b \geq 0$, the parameter constraints are

$$
\begin{array}{rl}
p_{2} \geq 0 & b \leq 2 s \\
p_{3} \geq 0 & \alpha \geq 0 \\
\epsilon \geq 0 & \alpha \geq \frac{s^{2}}{2 h-1+(b-2 s) / 2 s} .
\end{array}
$$

There are some conditional constraints on the parameter $h$. If $\alpha$ is a free parameter, eq. (29) imposes the constraint on $h$ :

$$
h>1-\frac{b}{4 s} \quad \text { for any } \alpha .
$$


For some models the choice $\alpha=s^{2}$ is convenient since then $p_{2}$ and $p_{3}$ are equal in tile plane $z=0$. With this choice eq. (29) will impose an additional constraint on $h$ and we have

$$
h>\frac{3}{2}-\frac{b}{4 s} \quad \text { if } \alpha=s^{2} .
$$

From the definition of the $z$-cutoff of the disc we have the constraint

$$
h^{2}>\frac{2 s-b}{2 s} \quad \text { for any } \alpha, b \neq 2 s .
$$

Finally, requiring the energy to be positive above the $z=0$ plane at the $z$ cutoff imposes the constraint

$$
-s^{2}+\alpha\left(2 h-1+\frac{b-2 s}{2 s}\right)-(h-1)^{2} \alpha^{2} H^{2}>0 .
$$

For a particular model all of the constraining equations need to be examined to determine the operative parameter limits.

\section{SOME MODELS}

The models described below are infinite discs in general relativity $\left(c_{1}=0\right)$ or for $c_{1} \neq 0$, an infinite disc with spin density in an EinsteinCartan space. The fluid parameters are the same for both cases.

A. Parameter choice: $b=0, \alpha=s^{2}$

The operative constraint for $h$ is eq. (31) or (33) giving $h>\frac{3}{2}$. We will choose $h=2$. For this choice

$$
\begin{gathered}
s H==\frac{ \pm 1}{\sqrt{3}}, \quad \mu(z)=-s^{2} z^{2} \\
\exp [\mu(z)] \epsilon=s^{2}\left(1-s^{2} z^{2}\right), \\
\exp [\mu(z)] p_{1}=s^{2}\left(1+4 s^{2} z^{2}\right), \\
\exp [\mu(z)] p_{2}=s^{2}\left(1-3 s^{2} z^{2}\right), \\
\exp [\mu(z)] p_{3}=s^{2}\left(1+4 s^{2} z^{2}\right) .
\end{gathered}
$$

In the $z=0$ plane the pressures are isotropic and the equation of state is $\epsilon=p$. 
The Einstein-Cartan spin density (and the equal vorticity $\omega_{r \phi}$ ) is

$$
S_{r \phi}=\exp \left[s^{2} z^{2}\right] c_{1} g r^{g-1}
$$

B. Parameter choice: $b=s, \alpha=s^{2}$

Equation (33) imposes the operative constraint on $h . h>1.25$. We will choose $h=2$ again. For this choice

$$
\begin{gathered}
s H=\frac{ \pm 1}{\sqrt{7}} \quad \text { and } \quad \mu(z)=-\frac{3 s^{2} z^{2}}{2}, \\
\exp [\mu(z)]\left(r+r_{0}\right)^{s} \epsilon=s^{2}\left(\frac{3}{2}-s^{2} z^{2}\right) \\
\exp [\mu(z)]\left(r+r_{0}\right)^{s} p_{1}=s^{2}\left(\frac{3}{2}+\frac{9 s^{2} z^{2}}{2}\right), \\
\exp [\mu(z)]\left(r+r_{0}\right)^{s} p_{2}=s^{2}\left(\frac{1}{2}-\frac{7 s^{2} z^{2}}{2}\right), \\
\exp [\mu(z)]\left(r+r_{0}\right)^{s} p_{3}=s^{2}\left(\frac{1}{2}+4 s^{2} z^{2}\right)
\end{gathered}
$$

Note that for this model in the $z=0$ plane there are equations of state

$$
p_{2,3}=\frac{\epsilon}{3} \quad \text { and } \quad p_{1}=\epsilon
$$

The spin density (and the equal vorticity) is the same as in case A.

Emphasizing again that the interior spin-vorticity content is in an Einstein-Cartan spacetime with the same fluid parameters as the static general relativistic spacetime, we plot graphs of the size of the vorticity vector (or spin vector) for both of the numerical examples given above. This will help clarify the role of the parameter $r_{0}$. The vorticity vector is

$$
\omega^{z}=e^{z 0 r \phi} \frac{\sqrt{f} \omega_{r \phi}}{D \exp [\mu+\beta / 2]}
$$

and $\Omega=\sqrt{\omega^{z} \omega_{z}}$. The size is shown as a function of radius in Figures la,b for the parameter choice $B$ discussed above. It is clear from these examples that $r_{0}$ parameterizes the location of the peak in the vorticity vector curve. 


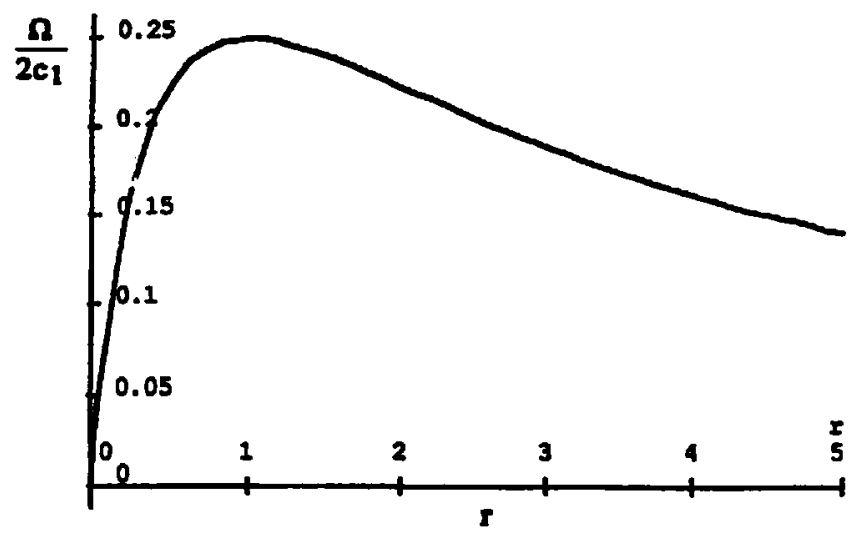

Figure 1a. Graph of $\Omega / 2 c_{1}$, versus radial distance $r$, in the $z=0$ plane for $r_{0}=1$, $b=s=2, g=2$.

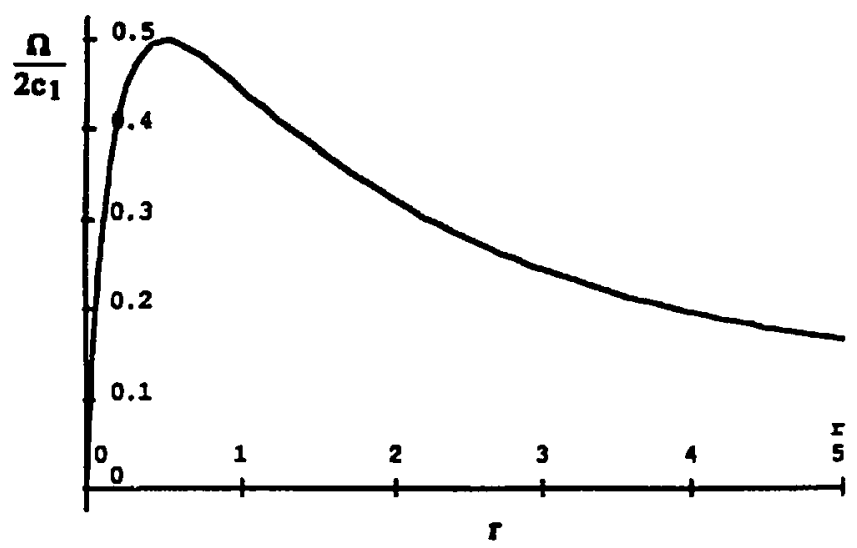

Figure 1b. Graph of $\Omega / 2 c_{1}$, versus radial distance $r$, in the $z=0$ plane for $r_{0}=0.5$, $b=s=g=2$.

\section{COMPARISON TO WALL SOLUTIONS}

The metric (16) is similar in structure to the general metric used to describe static general relativistic thin axis-symmetric walls $[4,20,26,27]$. As given in Ref. 4, the metric is

$$
\begin{aligned}
d s^{2}= & -r^{2 n} \exp [N] d t^{2}+r^{2 \kappa} \exp [2 P-N] d \phi^{2} \\
& +r^{2(\kappa-1)} \exp [Z-N]\left(d r^{2}+r^{2} d z^{2}\right)
\end{aligned}
$$


where $\kappa, n$ are constants, $N, P, Z$ are functions of $\theta$ and we have used $z$ instead of $\theta$.

Comparing with (16), we see that eq. (40) is equivalent to (16) with

$$
n=0, \kappa=s, \quad b=2 s, \quad N=h \alpha z^{2}, \quad P=\frac{\alpha z^{2}}{2}, \quad Z=0 .
$$

A classification of some wall solutions has been given by Lemos and Ventura [20]. While our metric is different from the ones that they classify, we note that for this choice of parameters $(n=0)$, the metric (16) lies at the origin of their classification diagram.

The fluid parameters generated by this choice of functions are easily found from (17)-(20) and one finds both pressures and tensions as is common in defect solutions. It is possible to match the metric (16) with the parameter choice given by (41) to a vacuum. The vacuum match at $z= \pm H$ is then

$$
d s^{2}=-d t^{2}+\left(r+r_{0}\right)^{2 s-2}\left(d r^{2}+z^{2}\left(r+r_{0}\right)^{2} d \phi^{2}\right)+d z^{2} .
$$

This match identifies the boundary $z^{2}=H^{2}=\alpha^{-1}$ and forces the interior fluid parameters $p_{2}=p_{3}=0, p_{1}=-\epsilon=s^{2}+\alpha+\alpha^{2} z^{2}$, a positive pressure and negative energy density. In general relativity, negative energy densities are unphysical. The possible physical acceptability of solutions with negative energy density in a spacetime with torsion, i.e. RiemannCartan spacetimes, have been discussed previously [24]. The strong energy condition may be satisfied in such spacetimes if an effective stress energy tensor is used in interpreting the energy conditions.

\section{DISCUSSION}

We have presented a solution to the gravitational field equations which describes either a static disc in general relativity or a spin polarized rotating disc in a Riemann-Cartan spacetime. The solution has many nice features: finite energy and pressure, a well defined $z$-boundary which is useful for modeling, and for some parameter choices, an isotropic pressure in the $z=0$ plane. It allows the modeling of a polarized rotating structure. It has a zero Riemann-Cartan vorticity but non-zero fluid vorticity and spin. While it is strictly non quantum-mechanical, in order to understand the relation between the spin and fluid vorticity of the medium in the disc one could draw a very qualitative analogy between the spin-vorticity structure of the fluid and the $J=0$ (with non-zero $L$ and $S$ ) states of some atomic systems. One might also make a qualititative analogy between 
counter rotating components and two different and oppositely polarized sources of angular momentum. Interesting questions can be asked about the spin polarization of the matter content of a specific galaxy, or, regarding a galaxy as a prime constituent, about the spin polarization of a galactic fluid. Some authors have also considered evidence [28] for a polarized universe and discussed some of the possible tests that one could make for this effect $[29,30]$. These questions are clearly of great importance in understanding the evolution of the angular momentum structure of galaxies and larger rotating systems.

\section{REFERENCES}

1. Oort, J. H. (1932). Bull. Astron. Netherl. 6, 249.

2. Friedman, A. M., Polyachenko, V. L. (1984). The Physics of Gravitating Systems. $I$ (Springer-Verlag, Berlin).

3. Goldreich, P., and Lynden-Bell, D. (1965). Mon. Not. R. Astr. Soc. 130, 97; (1965). ibid. 130, 125.

4. Lynden-Bell, D., and Pineault, S. (1978). Mon. Not. R. Astr. Soc. 185, 679; (1978). ibid. 185, 695.

5. Lemos, J. P. S. ( 1988). Mon. Not. R. Astr. Soc. 230, 451; id. (1989). Class. Quant. Grav. 6, 1219.

6. Morgan, T., and Morgan, L. (1969). Phys. Rev. 183, 1097; id. (1978). Phys. Rev. $D 2,2756$.

7. Bičak, J., Lynden-Bell, D., and Katz, J. (1993). Phys. Rev. D47, 4334.

8. Bičak, J., and Ledvinka, T. (1993). Phys. Rev. Lett. 71, 1669.

9. Kuzmin, G. G. (1956). Astron. Zh. 33, 27.

10. Evans, N. W., and deZeeuw, P. T. (1992). Mon. Not. R. Astr. Soc. 257, 152.

11. Krisch, J., and Smalley, L. S. (1993). Class. Quant. Grav. 10, 995.

12. Krisch, J., and Smalley, L. S. (1995). Gen. Rel. Grav. 27, 247.

13. Israel, W. (1966). Nuovo Cimento 44, 1.

14. Israel, W. (1970). Phys. Rev. D2, 641.

15. Voorhees, B. H. (1972). Phys. Rev. D5, 2413.

16. Lopez, C. A. (1981). Nuovo Cimento 66, 17.

17. Hamity, V. H., and Lamberti, P. W. (1987). Gen. Rel. Grav. 19, 917. Lamberti, P. W., and Hamity, V. H. (1989). Gen. Rel. Grav. 21, 869.

18. Bičak, J., and Ledvinka, T. (1993). Phys. Rev. Lett. 71, 1669.

19. Vilenkin, A. (1981). Phys. Rev. D23, 852.

20. Lemos, J. P. S., and Ventura, O. S. (1994). J. Math. Phys. 35, 3604.

21. Widrow, L. W. (1989). Phys. Rev. D39, 3571.

22. Dolgov, A. D., and Khriplovich, I. B. (1989). Gen. Rel. Grav. 21, 13.

23. Tomita, K. (1985). Phys. Lett. B162, 287; preprint RITP RRK 90-2.

24. Smalley, L. S., and Krisch, J. P. (1994). Phys. Lett. A196, 147.

25. Ray, J., and Smalley, L. S. (1982). Phys. Rev. Lett. 49, 1059; Phys. Rev. Lett. 50, 626(E); (1983). Phys. Rev. D27, 1383.

26. Lemos, J. P. S., and Letelier, P. S. (1991). Phys. Lett. A153, 288.

27. Lemos, J. P. S., and Letelier, P. S. (1993). Gen. Rel. Grav. 25, 365.

28. Birch, P. (1982). Nature 298, 451.

29. Rosquist, K. (1980). Gen. Rel. Grav. 12, 649

30. Obukov, Yu. iv. (1992). Gen. Rel. Grav. 24, 121. 